Revista del Centro de Investigación de la Universidad La Salle

Vol. 14, No. 53, Enero-Junio, 2020: 133-158

DOI: http://doi.org/10.26457/recein.v14i53.2688

\title{
COVID-19: fisiopatología y propuestas terapéuticas en investigación clínica
}

\section{COVID-19: pathophysiology and therapeutic proposals in clinical research}

\author{
Gilberto Guzmán Valdivia Gómez \\ Universidad La Salle México (México) \\ Alejandro Daniel Domínguez González \\ Universidad La Salle México (México) \\ Santiago Álvarez Rodríguez \\ Universidad La Salle México (México) \\ Dulce María Meneses Ruiz ${ }^{1}$ \\ Universidad La Salle México (México)
}

Recibido: 06 de junio de 2020

Aceptado: 12 de agosto de 2020

Publicado: 21 de septiembre de 2020

\section{Resumen}

La pandemia por COVID-19 declarada por la Organización Mundial de la Salud el 11 de marzo del 2020, ha ocasionado al momento 14,655,405 casos y 609,198 muertes en todo el

\footnotetext{
${ }^{1}$ Email: dulce.meneses@1asalle.mx
}

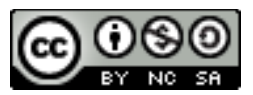

Revista del Centro de Investigación. Universidad La Salle por Dirección de Posgrado e Investigación. Universidad La Salle Ciudad de México se distribuye bajo una Licencia Creative Commons Atribución- 
Guzmán Valdivia Gómez, G.; Domínguez González, A. D.; Álvarez Rodríguez, S.; Meneses Ruiz, D. M.

mundo. Alrededor del $80 \%$ de las personas infectadas por SARS-CoV-2 son asintomáticos, el resto desarrolla la enfermedad COVID-19. Factores como edad, sexo y coexistencia de comorbilidades como hipertensión, diabetes y enfermedades cardiovasculares y del sistema respiratorio aumentan la gravedad de la enfermedad. Cuando la infección por SARS-CoV-2 alcanza el tracto respiratorio inferior, la respuesta inmune se acompaña de secreción intensa de citocinas y quimiocinas, dando lugar a edema y pérdida de la función de la unidad alveolocapilar que sumado a la tormenta de citocinas da lugar a la falla orgánica múltiple. Esta revisión describe la fisiopatología relacionada con las manifestaciones clínicas del COVID19 y presenta los principales fármacos antivirales e inmunomoduladores estudiados en ensayos clínicos.

Palabras clave: SARS-CoV-2, fisiopatología, COVID-19, perspectivas terapéuticas, reposicionamiento de fármacos 


\section{Abstract}

The COVID-19 pandemic declared by the World Health Organization on March 11, 2020, has currently caused $14,655,405$ cases and 609,198 deaths worldwide. About $80 \%$ of people infected with SARS-CoV-2 are asymptomatic, the rest develop COVID-19 disease. Factors such as age, sex, and coexistence of comorbidities such as hypertension, diabetes, and cardiovascular and respiratory system diseases increase the severity of the disease. When SARS-CoV-2 infection reaches the lower respiratory tract, the immune response is accompanied by intense secretion of cytokines and chemokines, leading to edema and loss of function of the alveolar-capillary unit that added to the cytokine storm it gives rise to multiple organic failure. This review describes the pathophysiology related to the clinical manifestations of COVID-19 and presents the main antiviral and immunomodulatory drugs studied in clinical trials.

Keywords: SARS-CoV-2, pathophysiology, COVID-19, therapeutic perspectives, drug repositioning 
Guzmán Valdivia Gómez, G.; Domínguez González, A. D.; Álvarez Rodríguez, S.; Meneses Ruiz, D. M.

\section{Introducción}

Los coronavirus (CoVs) son considerados importantes patógenos para los humanos. En 2002 en Guangdong, China, se presentó el primer brote por SARS-CoV que produce el síndrome respiratorio agudo grave (SARS), éste ocasionó 8422 casos, 916 (10.8\%) de ellos fatales en 29 países de los seis continentes. Para 2012 se presentó un brote por CoVs en Arabia Saudita ocasionando el síndrome respiratorio de Oriente Medio (MERS-CoV), que ocasionó más de 2500 casos con una tasa de mortalidad de 34.4\%, convirtiendo al MERS-CoV en uno de los virus más mortales para la especie humana (Park et al., 2020).

En diciembre de 2019, en Wuhan China se presentaron una serie de casos de neumonía causados por un nuevo CoVs cuya enfermedad fue denominada por la Organización Mundial de la Salud como enfermedad del coronavirus 2019 (COVID-19, por sus siglas en inglés) (Coronaviridae Study Group of the International Committee on Taxonomy of Viruses, 2020), posteriormente, el Comité Internacional de Taxonomía de Virus designó a este virus como SARS-CoV-2. La rápida diseminación de la enfermedad condujo a que la OMS declarara el 11 de marzo de 2020 a COVID-19 como pandemia (Liu et al., 2020).

Aun cuando la mayoría de los pacientes infectados por SARS-CoV-2 no presentan síntomas o desarrollan síntomas leves, desde el reporte del primer caso hasta mediados de julio de 2020 se han registrado 14,655,405 casos confirmados e incluyendo 609,198 muertes por COVID-19 a nivel mundial. En México, se han reportado 349,396 casos confirmados y 39,485 muertes (Organización Mundial de la Salud, 2020).

Es ampliamente conocido que los CoVs infectan animales salvajes como aves, murciélagos, ratones, jirafas y ballenas. Además, que animales domésticos pueden participar como hospederos intermediarios, permitiendo su transmisión desde sus hospederos naturales a los humanos (Cui et al., 2019; Hasöksüz et al., 2020; Zhang et al., 2007). Cabe esperar que nuevos CoVs emerjan periódicamente entre los humanos por la recombinación frecuente del genoma viral, su alta prevalencia, la capacidad de infectar diferentes especies y las condiciones de interacción humano-animal (Hasöksüz et al., 2020). 
Esta investigación describe de modo claro el estado del conocimiento sobre la fisiopatología de las manifestaciones clínicas de la neumonitis y de la afectación multisistémica en COVID-19 y los recursos terapéuticos que se están aplicando para contener los efectos de la enfermedad.

\section{Coronavirus SARS-CoV2}

El SARS-CoV-2 es un virus esférico, con envoltura compuesta por una bicapa lipídica, con diámetro aproximado de 70 a $90 \mathrm{~nm}$ y un genoma compuesto de ARN monocatenario positivo de 30,000 pares de bases $(30 \mathrm{~kb})$ cuya secuencia genética tiene más de $80 \%$ de identidad con SARS-CoV y 50\% con MERS-CoV (Jin et al., 2020). Su genoma codifica cuatro proteínas estructurales: $\mathrm{S}$ (espícula), $\mathrm{E}$ (envoltura), $\mathrm{M}$ (membrana) y $\mathrm{N}$ (nucleocápside), de éstas, la glicoproteína S, expresada en la superficie del virión y que le confiere la apariencia de corona, es la que posee el sitio de reconocimiento al receptor celular del hospedero. Esta glicoproteína conformada por dos subunidades, la S1 (expuesta) que constituye el $70 \%$ de la proteína y comparte el $70 \%$ de su secuencia con el SARS del murciélago y el pangolín, y la subunidad S2 (transmembranal) con identidad del $99 \%$ con otros SARS. La subunidad S1 del SARS-CoV-2 posee un dominio que interacciona con la enzima convertidora de angiotensina 2 (ECA2), proteína integral de membrana que funciona como receptor y sitio de internalización del virus. ECA2 se expresa en células de la mucosa orofaríngea, pulmón (principalmente en neumocitos tipo II), sistema cardiovascular, riñón e intestino, principalmente (Walls et al., 2020).

\section{Enfermedad COVID-19}

Las vías de transmisión del SARS-CoV-2 son por gotas de secreciones respiratorias y contacto de persona a persona a través de fomites. Recientemente, los investigadores australianos Morawska y Milton, apoyados por otros 239 científicos de diferentes partes del mundo, reconocen el potencial de propagación del virus SARS-CoV-2 a través de microgotas en aerosol lo suficientemente pequeñas como para permanecer suspendidas en el aire y presentar un riesgo de exposición a distancias mayores de uno a dos metros de un individuo infectado. Este problema es especialmente grave en ambientes cerrados en los que hay 
Guzmán Valdivia Gómez, G.; Domínguez González, A. D.; Álvarez Rodríguez, S.; Meneses Ruiz, D. M.

muchas personas y con ventilación inadecuada (Morawska y Cao, 2020). Se han propuesto otras posibles vías de transmisión como la vertical (materno-fetal), lactancia materna y fecaloral, sin que al momento exista evidencia científica concluyente (Bulut y Kato, 2020; KarimiZarchi et al., 2020).

El período de incubación de SARS-CoV-2, considerado el tiempo que transcurre entre la exposición al virus y el inicio de los síntomas, es de 5.2 días, pero pueden transcurrir hasta dos semanas. La presentación clínica de los individuos infectados por SARS-CoV-2 es muy variada, la mayoría de los individuos permanece asintomático (80 \%) y el resto desarrolla la enfermedad denominada COVID-19, en la que los síntomas más comunes son fiebre (86 a $97 \%$ ), tos (59 a $76 \%$ ), astenia (34 a $68 \%$ ), disnea (21 a $40 \%$ ), diarrea (20 a 40 $\%$ ) y en menor proporción hemoptisis, vómito y cefalea. Estudios observacionales reportan aumento de complicaciones clínicas por COVID-19 en relación con la prevalencia de comorbilidades como hipertensión (13 a $27.2 \%$ ), diabetes (7.2 a $12.2 \%$ ) y enfermedades cardiovasculares (3.8 a 13.8) y del sistema respiratorio (0.9 a $2.1 \%$ ) (Yang J, et al., 2020). Los adultos mayores y las personas con inmunosupresión pueden presentar síntomas neurológicos "atípicos" como fatiga, disminución del estado de alerta, movilidad reducida, diarrea, anorexia y delirio, en ausencia de fiebre (McMichael et al., 2020; Tay y Harwood, 2020).

Las manifestaciones documentadas en pacientes con COVID-19 afectan primordialmente el tracto respiratorio, pero también se ha documentado la afección de otros órganos y sistemas con prevalencias que varían de acuerdo a la presentación clínica e incluyen enfermedades cardiacas (8.9 a $52 \%$ ), gastrointestinales (9.2 a $26.8 \%$ ), hepáticas (16.1 a $53.1 \%)$, renales $(0.5$ a $23 \%)$, neurológicas $(0.5$ a $16.8 \%)$, oculares $(1.1$ a $15.9 \%)$, cutáneas (1.1 a $15.9 \%)$ y hematológicas con linfopenia $(56.5 \%)$ y tromobocitopenia (16.4 a $32.3 \%$ ). Ocasionalmente estos signos y síntomas se presentan al inicio de la infección por SARS-CoV-2, antes que la fiebre o las manifestaciones respiratorias (Lai et al., 2020).

De acuerdo a la presentación clínica, la enfermedad se ha dividido en tres fases. La fase I, llamada de infección temprana, ocurre durante los primeros siete a diez días después del contagio y se caracteriza por presentar síntomas leves. La fase II, llamada fase pulmonar, se manifiesta en los siguientes siete a diez días y se subdivide en una fase inicial IIa, 
caracterizada por fiebre, tos productiva, taquipnea y dificultad respiratoria leve; se presenta afectación en el parénquima pulmonar, observándose infiltrados diseminados en las imágenes de tórax; en la fase IIb hay datos francos de hipoxia, tos productiva e incremento en la dificultad respiratoria, se presenta un proceso inflamatorio pulmonar severo con edema pulmonar y saturación de oxígeno por debajo del 90\%; a partir de esta fase el paciente requiere manejo intrahospitalario. La fase III, llamada de hiperinflamación, presenta los síntomas secundarios a la afectación multisistémica (Siddiqi y Mehra, 2020).

\section{Fisiopatología de COVID-19}

La fijación del virus SARS-CoV-2 a la célula huésped se da por la unión de la subunidad 1 de la proteína $\mathrm{S}$ del virus a las proteínas de membrana ECA2 (enzima) y a la glicoproteína CD147 (inmunomodulador) de la célula. Esta fijación se fortalece con la acción de la proteína M del coronavirus. En la membrana celular, este complejo de virus-receptor activa la proteína Serina Proteasa Transmembrana (TMPRS, por sus siglas en inglés) que rompe la integridad de la membrana celular permitiendo la adsorción del virus junto con el receptor ECA2. En el ribosoma y aparato de Golgi se lleva a cabo el proceso de replicación viral. El virus se une a receptores de la membrana nuclear llamados importinas y penetra al núcleo a través de los complejos de poros nucleares. El virus SARS-CoV-2 retarda la acción del interferón 1, disminuyendo su respuesta antiviral y en consecuencia aumentando la infección. Además, el SARS-CoV-2 disminuye el número de receptores ECA2, reduciendo considerablemente la conversión de angiotensina 2 (vasoconstrictor potente) en angiotensina 1-9 y angiotensina 1-7, esta última con efectos antinflamatorios, antifibróticos y antioxidantes (Vaduganathan et al., 2020).

La infección por SARS-CoV-2 inicia en el tracto respiratorio superior, donde la replicación viral en células epiteliales de naso y orofaringe conduce a la activación de la respuesta inmune innata y a la resolución de la infección en los pacientes asintomáticos (Fu et al., 2020). En los pacientes susceptibles, la respuesta inmune innata es ineficiente y la infección alcanza el tracto respiratorio inferior. A nivel alveolar, la infección induce la activación de las células epiteliales, endotelio, macrófagos y células dendríticas, que secretan citocinas (IFN $\alpha$ y $\beta$, IL-1, IL-6, TNFa) y quimiocinas (IL-8, CCL-2,3 y 5), que potencian la respuesta inmune innata inicial e incrementan la permeabilidad capilar produciendo edema e 
Guzmán Valdivia Gómez, G.; Domínguez González, A. D.; Álvarez Rodríguez, S.; Meneses Ruiz, D. M.

infiltración de células inflamatorias en el alveolo pulmonar, causando pérdida de función de neumocitos tipo II, disminución de la secreción del factor surfactante, colapso alveolar y formación de una membrana hialina, comprometiendo el intercambio gaseoso y dando lugar al Síndrome de Dificultad Respiratoria Aguda (SDRA), manifestándose clínicamente con tos, fiebre, disnea y cianosis (Fu, et al, 2020) (Figura 1).

Se ha documentado que la infección por SARS-CoV-2 suprime la respuesta inmune al inhibir la señalización del interferón 1 (IFN $\alpha$ y $\beta$ ), que inicialmente tiene un efecto antiviral (Channappanavar et al., 2016). Aproximadamente del 5 al $10 \%$ de los pacientes infectados por SARS-CoV-2 desarrollan consolidación pulmonar (neumonía) acompañada de una respuesta inmunológica exagerada conocida como "tormenta de citocinas". Se propone que esta respuesta está relacionada con diversos factores como el antígeno de histocompatibilidad de linfocitos, carga viral infectiva, la capacidad del virus de evadir la respuesta inmune, respuesta tardía de los interferones alfa y beta, y respuesta inmune deficiente en pacientes con enfermedades crónicas; en estos pacientes, la "tormenta de citocinas" conlleva complicaciones sistémicas (Figura 1) (Ye et al., 2020). 


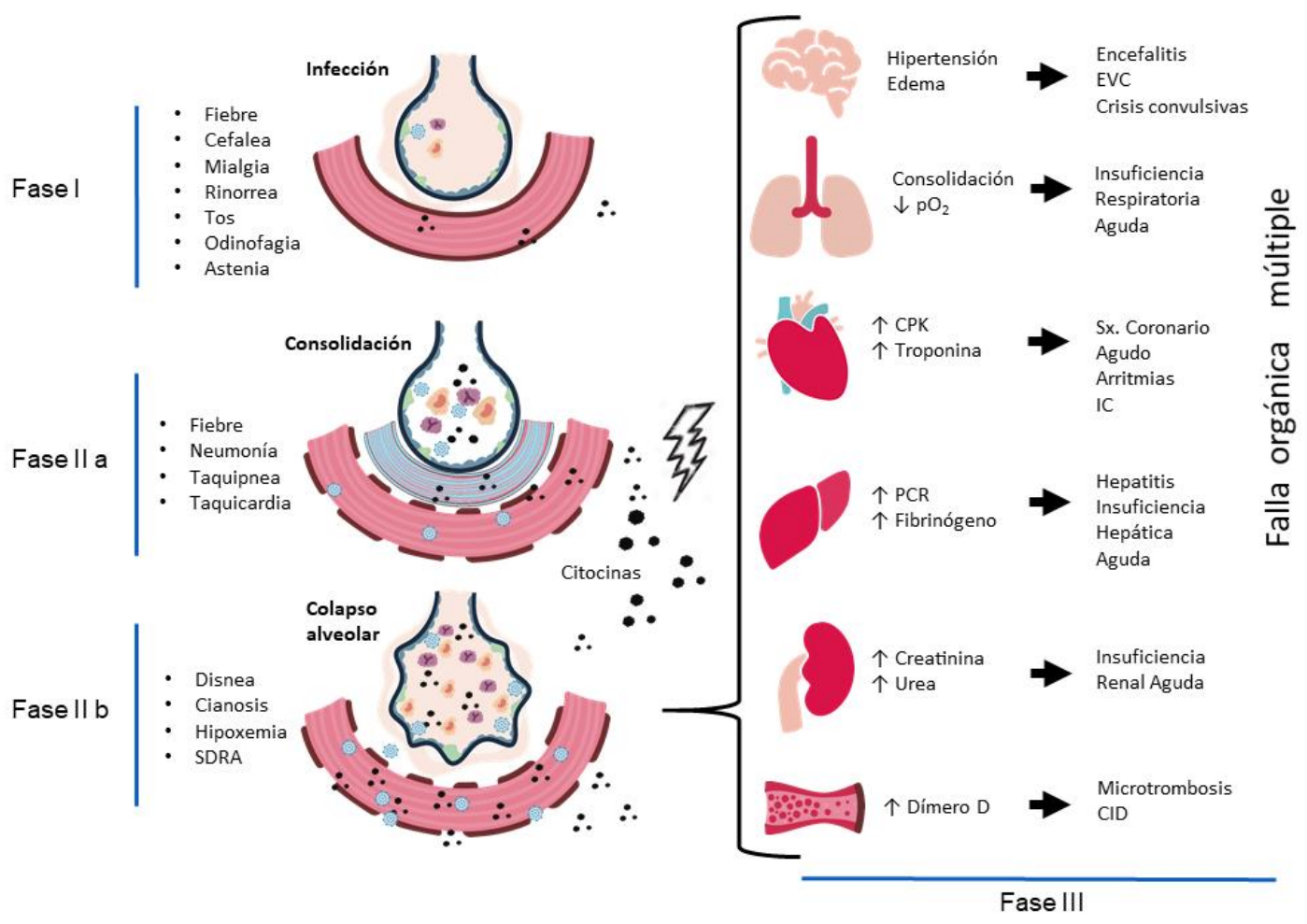

Figura 1. Fisiopatología de la enfermedad COVID-19 y sus manifestaciones clínicas pulmonares y sistémicas. El virus SARS-CoV-2 ingresa generalmente por la vía respiratoria y llega al alveolo pulmonar por ventilación. La infección y replicación viral genera secreción de citocinas y quimiocinas por células endoteliales e inmunes, provocando incremento de permeabilidad vascular e infiltración inflamatoria. Esto da lugar a la aparición de fiebre, mialgia, astenia y tos, los primeros síntomas de la enfermedad. Cuando no se logra contener la infección, se produce consolidación alveolar que compromete el intercambio gaseoso e incremento sustancial de mediadores inflamatorios, dando lugar a la liberación masiva de citocinas al torrente sanguíneo, conocida como "tormenta de citocinas". La activación del endotelio vascular por la respuesta sistémica y la infección viral activa la cascada de coagulación provocando la formación de microtrombos en capilares de diversos órganos que aunado a la vasodilatación ocasiona falla orgánica múltiple. Abreviaturas: CID, coagulación intravascular diseminada; CPK, creatinfosfoquinasa; EVC, Evento Vascular Cerebral; IC, insuficiencia cardiaca; IL, interleucina; $\mathrm{PCR}$, proteína $\mathrm{C}$ reactiva; $\mathrm{pO}_{2}$, presión parcial de oxígeno; $\mathrm{Sx}$., síndrome; TNF, factor de necrosis tumoral.

La tormenta de citocinas y la infección de células endoteliales por SARS-CoV-2 activan la cascada de la coagulación sanguínea provocando la formación de microtrombos tanto en territorio venoso como arterial, que afectan a la mayoría de los órganos, principalmente cerebro, corazón, pulmones, hígado y riñones, con la consecuente alteración en sus funciones, provocando falla orgánica múltiple y la muerte en más del 50\% de los pacientes (Ye et al., 2020). Esta activación generalizada de la cascada de la coagulación, con la formación inicial de microtrombos y la subsecuente activación del sistema fibrinolítico son los responsables 
Guzmán Valdivia Gómez, G.; Domínguez González, A. D.; Álvarez Rodríguez, S.; Meneses Ruiz, D. M.

de la elevación del fibrinógeno y dímero $\mathrm{D}$, que han sido catalogados como indicadores de gravedad (Prete et al., 2020).

Las complicaciones más comunes que se desarrollan en COVID-19 son neumonía bilateral, que puede progresar a SDRA, choque séptico, daño renal y cardiaco agudos (arritmias, insuficiencia cardíaca, infarto agudo del miocardio), coagulopatía, rabdomiólisis, hiponatremia y acidosis (Prete et al., 2020). En la cohorte de estudio de 1099 casos de COVID-19 (Guan et al., 2020), la neumonía bilateral se presentó en 91,1\% de los casos, seguido en frecuencia de SDRA (3,4\%), choque séptico (1,1\%), Insuficiencia Renal Aguda $(0,5 \%)$ y coagulopatía $(0,1 \%)$. En esta cohorte, $926(84 \%)$ pacientes presentaron enfermedad no grave y $173(16 \%)$ enfermedad grave.

Los predictores independientes de alta mortalidad para el COVID-19 son la edad avanzada (mayor de 70 años); comorbilidades subyacentes tales como hipertensión no controlada, diabetes, enfermedad coronaria, enfermedad pulmonar obstructiva crónica y neoplasias malignas. Los factores citoquímicos de mal pronóstico son: linfopenia grave $\left(<0.810^{9} / \mathrm{L}\right)$ y elevación del dímero $\mathrm{D}(>1 \mu \mathrm{g} / \mathrm{L})$, de la proteína $\mathrm{C}$ reactiva, deshidrogenasa láctica (DHL), alanina aminotransferasa (ALT), ferritina sérica, IL-6 y troponina cardíaca de alta sensibilidad (Chen et al., 2020).

\section{Aproximaciones terapéuticas farmacológicas contra el SARS-CoV-2}

Debido a que no se han reportado fármacos específicos para el tratamiento de COVID-19 por ser una enfermedad emergente, las aproximaciones terapéuticas empleadas hasta el momento son medicamentos que han sido utilizados para otras enfermedades y que por sus mecanismos de acción podrían ser eficaces. Esta medida se conoce como reposicionamiento de fármacos, una estrategia esencial y universal que reduce el curso de los ensayos clínicos (fases clínicas I y II) y el costo de producción y distribución de los medicamentos por la preexistencia de cadenas de suministro farmacéutico, además de facilitar el diseño in silico de nuevos medicamentos. (Rosa y Santos, 2020).

En el caso de COVID-19, los fármacos reposicionados para el tratamiento están relacionados principalmente con los utilizados en enfermedades virales como MERS, SARS 
e influenza, así como para estados de hiperinflamación que presentan estados fisiopatológicos similares al COVID-19 como artritis reumatoide, lupus eritematoso y linfohistiocitosis hemofagocítica secundaria, entre otros.

Se están probando fármacos que interfieren en diferentes etapas del ciclo de infección viral como Cloroquina (Zhou et al., 2020) e Hidrocloroquina (Sanders et al., 2020; Zhou et al., 2020) en la adsorción del virus, y con Remdesivir (Wang et al., 2020) y la combinación Lopinavir/ Ritonavir (Cao et al., 2020; Sanders et al., 2020) en la replicación y síntesis viral, fármacos que se utilizan en todas las fases clínicas de la enfermedad. En el manejo de la respuesta inflamatoria se están probando inmunomoduladores como Anakinra (Mehta et al., 2020) y Rilonacept (Liu et al., 2020), que regulan la expresión de IL-1; anticuerpos monoclonales contra IL-6 como Tocilizumab (Xu et al., 2020; Zhang et al., 2020) y Sarilumab (Ranieri et al., 2020) e Interferón 1 ( $\alpha$ y $\beta$ ) (Ranieri et al., 2020) en pacientes hospitalizados, es decir, a partir de la fase IIb de la enfermedad (Figura 2).

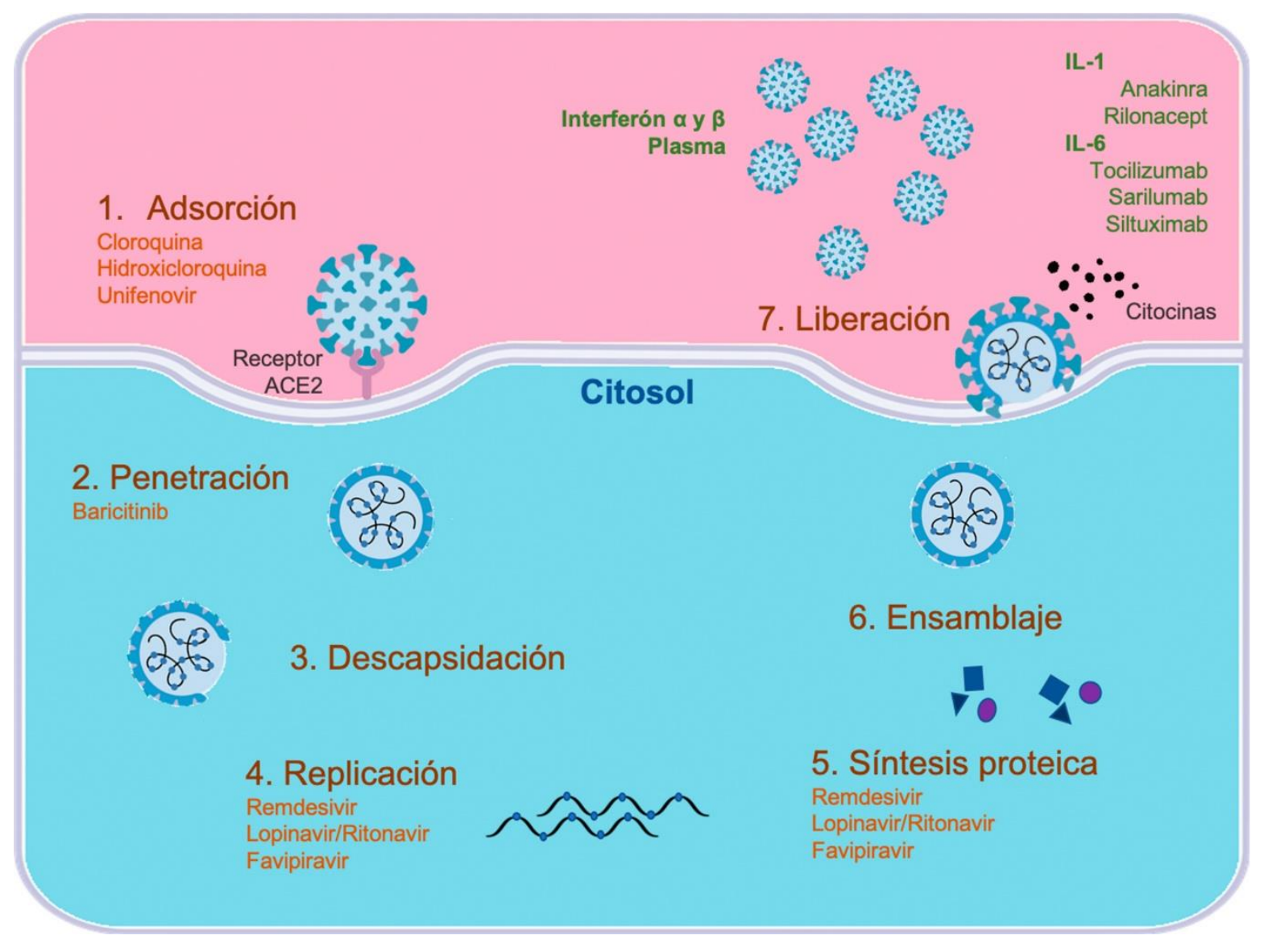


Guzmán Valdivia Gómez, G.; Domínguez González, A. D.; Álvarez Rodríguez, S.; Meneses Ruiz, D. M.

Figura 2. Ciclo de replicación del SARS-CoV-2 y sitios de acción de algunos medicamentos. Este esquema muestra las diferentes fases del ciclo viral, desde la fijación al receptor ACE2, penetración, replicación, síntesis, ensamblaje de las proteínas estructurales y no estructurales y liberación de miles de virus; así como los principales medicamentos que se encuentran bajo investigación en ensayos clínicos actuales.

También se está estudiando la eficacia y seguridad de medicamentos dirigidos a las complicaciones de la fase de hiperinflamación del COVID-19 que incluyen glucocorticoides sintéticos (dexametasona) (Ledford, 2020), anticoagulantes a dosis profiláctica y terapéutica, y medicamentos propios para el mantenimiento adecuado del funcionamiento de los órganos afectados, estos últimos no evaluadas en el presente artículo.

En la Tabla 1 se muestran los principales medicamentos con propiedades antivirales y los dirigidos a la hiperinflamación sistémica, que se encuentran en diferentes etapas de investigación clínica para el tratamiento del COVID-19 en México y el mundo. La mayoría de los fármacos están aprobados para otras enfermedades por la Administración de Alimentos y Medicamentos (FDA) de los Estados Unidos, ninguno específicamente para COVID-19. Otros medicamentos incluidos en la tabla que, aunque no están aprobados por la FDA, son fármacos que actualmente se encuentran en estudio y cuyos resultados podrán posicionarlos como eficaces y seguros.

\section{Tabla 1.}

Medicamentos antivirales e inmunomoduladores que se están estudiando en ensayos clínicos para el tratamiento de COVID-19

\begin{tabular}{llll}
\hline $\begin{array}{c}\text { Tratamientos } \\
\text { antivirales }\end{array}$ & Probables mecanismos de acción & Aprobación FDA & $\begin{array}{c}\text { Ensayos clínicos en el mundo y } \\
\text { México }\end{array}$ \\
\hline Azitromicina & $\begin{array}{l}\text { Induce expresión de genes } \\
\text { estimulados por interferón, } \\
\text { atenuando la replicación viral }\end{array}$ & $\begin{array}{l}\text { Infección por } \\
\text { micobacterias no } \\
\text { tuberculosas }\end{array}$ & $\begin{array}{l}\text { 104 estudios para el tratamiento de } \\
\text { COVID-19 solo o en combinación } \\
\text { con Hidroxicloroquina. }\end{array}$ \\
& Activa los polimorfonucleares & & \\
& $\begin{array}{l}\text { Reduce la secreción de citocinas } \\
\text { inflamatorias (IL-6 e IL-8) en las } \\
\text { células epiteliales y del factor de } \\
\text { crecimiento fibroblástico en las }\end{array}$ & $\begin{array}{l}\text { Infecciones de } \\
\text { transmisión } \\
\text { sexual y otras } \\
\text { infecciones }\end{array}$ & $\begin{array}{l}\text { Un estudio en México en ISSSTE, } \\
\text { CDMX asociado a Ivermectina y } \\
\text { Cloramfenicol. }\end{array}$ \\
& & &
\end{tabular}


células del músculo liso de las vías

respiratorias

(Zhou et al., 2020)

Baricitinib Inhibidor selectivo y reversible de la Janus quinasa (JAK1 y JAK2)

(Cantini et al., 2020)

Aumenta el $\mathrm{pH}$ de las vacuolas intracelulares y las destruye

Cloroquina

Altera las vías de degradación de proteínas, lo que interfiere con la fusión virus / célula

Inhibe la glicosilación del receptor celular ACE2, por lo que interfiere con la unión del virus al receptor celular

Reduce las citocinas proinflamatorias

(Zhou et al., 2020)

Favipiravir Inhibidor de la ARN polimerasa dependiente de ARN (RdRp)

(Cai et al., 2020)

Hidroxicloro - quina

Altera las vías de degradación de proteínas, lo que interfiere con la fusión virus / célula

Inhibe la glicosilación del receptor celular ACE2, por lo que interfiere con la unión del virus al receptor celular

Reduce la secreción de citocinas proinflamatorias
Artritis

reumatoide

14 ensayos clínicos en pacientes con enfermedad moderada y grave.

En México, estudio en pacientes graves por Lilly y compañía en Fase 3.

Malaria

Amibiasis extraintestinal

No aprobado por FDA

Aprobada en Japón para Influenza y en China para COVID-19

30 ensayos clínicos en pacientes hospitalizados graves.

Sin estudios en México.

Lupus

eritematoso

Malaria

209 Ensayos clínicos en pacientes hospitalizados, incluyen a embarazadas.

En México: Dos ensayos clínicos en el INER en Fase 3.

Ensayo asociado a Bromexina

Artritis reumatoide (mucolítico y expectorante) en INR en Fase 1.

Ensayo asociado a Nitasoxanida (antiparasitario, con efecto antiviral en estudio) en Hospital materno 
Guzmán Valdivia Gómez, G.; Domínguez González, A. D.; Álvarez Rodríguez, S.; Meneses Ruiz, D. M.

(Sanders et al., 2020; Zhou et al., 2020)

Ivermectina Inhibe la penetración del virus al núcleo al fijarse en proteína importina.

(Sharun et al., 2020)

Lopinavir/ Ritonavir

Nitasoxanida

Inhibe fijación del virus a la célula huésped.

Reduce la secreción de citocinas proinflamatorias

(Calderón et al., 2020)

Inhibe la ARN polimerasa dependiente de ARN viral

(Wang et al., 2020)
Antiparasitario

Infección por VIH

Antiprotozoarios

16 ensayos clínicos como medicamento aislado y en combinación con Ivermectina y Nitasoxanida.

En México dos ensayos, uno asociado con Hidroxicloroquyina en fase 4 y otro solo en Fase 4, ambos en el Hospital Materno Perinatal "Mónica Pretelini" del Estado de México.

No aprobado por la FDA

Agente antiviral en investigación
36 ensayos clínicos, algunos multicéntricos, en pacientes hospitalizados con enfermedad moderada y severa.

En México: Dos ensayos clínicos, uno en el Hospital Naval de Alta especialidad y en el Hospital General 


\begin{tabular}{lll}
\hline \hline Ruxolitinib & $\begin{array}{l}\text { Inhibidor selectivo y reversible de la } \\
\text { Janus quinasa (JAK1 y JAK2) }\end{array}$ & $\begin{array}{l}\text { Enfermedad } \\
\text { aguda injerto vs } \\
\text { huésped } \\
\text { refractaria a } \\
\text { esteroides }\end{array}$
\end{tabular}

de México "Dr. Eduardo Liceaga”, ambos en Fase 2.

19 ensayos clínicos en evidencia de tormenta de citocinas.

En México un ensayo clínico en Grupo Cooperativo de Hemopatías Malignas en Huixquilucan, Estado de México, Fase 1 y 2.
Umifenovir Inhibe la fusión del segmento S1 con el receptor de membrana de la célula

(Costanzo et al., 2020)
Influenza

9 ensayos clínicos en pacientes hospitalizados comparándolo con placebo, interferones y Lopinavir/Ritonavir.

Sin estudios en México.

Tratamientos
inmuno-
reguladores

\begin{tabular}{|c|c|c|c|}
\hline Anakinra & $\begin{array}{l}\text { Inhibidor de la IL-1 } \\
\text { (Mehta et al., 2020) }\end{array}$ & $\begin{array}{l}\text { Artritis } \\
\text { reumatoide }\end{array}$ & $\begin{array}{l}23 \text { ensayos clínicos en pacientes con } \\
\text { gran respuesta inflamatoria. } \\
\text { Sin estudios en México. }\end{array}$ \\
\hline Colchicina & $\begin{array}{l}\text { Inhibidor de interleucinas } \\
\text { (Corral et al., 2020) }\end{array}$ & $\begin{array}{l}\text { Brotes agudos de } \\
\text { Gota } \\
\text { Fiebre del } \\
\text { Mediterráneo } \\
\text { familiar. }\end{array}$ & $\begin{array}{l}15 \text { ensayos clínicos en pacientes } \\
\text { graves. } \\
\text { En México un ensayo clínico en el } \\
\text { INCMNSZ en Fase } 2 \text {. }\end{array}$ \\
\hline $\begin{array}{c}\text { Dexametaso } \\
\text { na }\end{array}$ & $\begin{array}{l}\text { Antinflamatorio } \\
\text { Inmunosupresor } \\
\text { (Villar et al., 2020) }\end{array}$ & $\begin{array}{l}\text { Antinflamatorio } \\
\text { Inmunomodulado } \\
\mathrm{r}\end{array}$ & $\begin{array}{l}18 \text { estudios en pacientes graves con } \\
\text { falla respiratoria aguda. }\end{array}$ \\
\hline $\begin{array}{l}\text { Interferones } \\
\qquad(\alpha \mathrm{y} \beta)\end{array}$ & $\begin{array}{l}\text { Inhiben la secreción de citoquinas. } \\
\text { Tiene cierto efecto antiviral por lo } \\
\text { que se está estudiando en las etapas } \\
\text { iniciales de la enfermedad, cuando } \\
\text { hay más carga viral } \\
\text { (Ranieri et al., 2020) }\end{array}$ & $\begin{array}{l}\text { IFN alfa-2b: } \\
\text { leucemia, } \\
\text { melanoma, } \\
\text { linfoma, } \\
\text { condilomas } \\
\text { acuminados, } \\
\text { sarcoma de }\end{array}$ & $\begin{array}{l}75 \text { ensayos clínicos en pacientes con } \\
\text { poca respuesta a otros tratamientos } \\
\text { principalmente en combinación con } \\
\text { antivirales. }\end{array}$ \\
\hline
\end{tabular}


Guzmán Valdivia Gómez, G.; Domínguez González, A. D.; Álvarez Rodríguez, S.; Meneses Ruiz, D. M.

Kaposi, hepatitis

$\mathrm{B}$, hepatitis $\mathrm{C}$

IFN alfa-1b no

está disponible en

los Estados

Unidos.

Esclerosis

múltiple (IFN

beta-1a, IFN

beta-1b)

Mavrilimum Anticuerpo monoclonal humano que $\mathrm{ab} \quad$ inhibe el receptor del factor estimulante de colonias de macrófagos de granulocitos humanos.

(De Luca et al, 2020)

Rilonacept Antagonista recombinante del receptor de IL-1 humano

(Liu et al., 2020)

Sarilumab Anticuerpo monoclonal activo contra el receptor de interleucina-6 (IL-6R)

(Ranieri et al., 2020; Sanofi, 2020)

Siltuximab Anticuerpo monoclonal quimérico humano-ratón recombinante que se une a IL-6, inhibiendo la señalización de IL-6

(Bonam et al., 2020)

Tocilizumab Anticuerpo monoclonal activo contra el receptor de interleucina-6 (IL-6R), también bloquea la respuesta inmune mediada por IL-6 en COVID-19

(Xu et al., 2020; Zhang et al., 2020)
No aprobada por

FDA

Artritis reumatoide

Artritis reumatoide

Enfermedad de Castelman

Artritis reumatoide

Otras artritis de origen autoinmune
4 ensayos clínicos en Fase II para COVID-19.

Sin estudios en México.

Un ensayo clínico en pacientes con gran respuesta inflamatoria.

Sin estudios en México.

17 ensayos clínicos en pacientes con gran respuesta inflamatoria, sola o en combinación con antivirales.

Sin estudios en México.

3 ensayos clínicos en pacientes con SDRA, uno comparándola con corticoesteroides.

Sin estudios en México.

59 ensayos clínicos en pacientes con gran respuesta inflamatoria.

En México: 1 ensayo clínico en el INC, Fase 2. 
Transfusión

de plasma de

pacientes

convalecient

es

\section{Estimula la citotoxicidad de}

linfocitos $\mathrm{T}$ mediada por anticuerpos

Estimula la fagocitosis del virus

(Zeng et al., 2020)
Contiene anticuerpos específicos

virus anti SARS-Cov-2 que neutralizan al
No aprobada por

la FDA

la

(a)

No se recomienda su uso de primera elección en pacientes con COVID19. Hay 309 ensayos clínicos con pacientes graves y críticos.

En México: nueve ensayos clínicos en hospitales públicos: Centenario Hospital Miguel Hidalgo en Aguascalientes, Fase 3, Centro de Hematología y Medicina Interna en Puebla, Fase 2, Hospital Del ISSSTE Regional en Guadalajara, Jalisco, Secretaria de Salud del Estado de Sonora, Hospital General del Estado en Hermosillo, Sonora, Fases 1 y 2 , Hospital Central Norte Pemex en CDMX, Fase 2 y privados: Hospital San José en Monterrey, Nuevo León, Fase 1; Hospital Naval de Alta Especialidad en CDMX y Hospital General de México "Dr. Eduardo Liceaga" en CDMX, en Fase 2, dos ensayos en el Hospital Universitario "Dr. Gonzalo Valdés Valdés" en Saltillo, Coahuila, Fase 1 y 2.

Vacuna para 18 ensayos clínicos. prevención de tuberculosis
En México ensayo clínico en el Hospital Dr. José Eleuterio González de la UANL en Fase 3.

Vacuna BCG Inmuno potenciador

Fuente. Elaboración propia con información de la plataforma clinicaltrials. gov como referencia en los estudios de investigación de medicamentos. Abreviaturas: ACE: Enzima Convertidora de Angiotensina; Ags.: Aguascalientes; ARN: Ácido ribonucleico; AZM: Azitromicina; BCG: Bacilo de Callmette-Guérin; CMN: Centro Médico Nacional; FDA: Food and Drug Administration; HCQ: Hidroxicloroquina; IFN: Interferón; IL: Interleucina; IMSS: Instituto Mexicano del Seguro Social; INC: Instituto Nacional de Cancerología; INCMNSZ: Instituto de Ciencias Médicas y Nutrición "Salvador Zubirán"; INER: Instituto Nacional de Enfermedades Respiratorias; INR: Instituto Nacional de Rehabilitación; ISSSTE: Instituto de Seguridad y Servicios Sociales de los Trabajadores del Estado; SDRA: Síndrome de Dificultad Respiratoria Aguda; UANL: Universidad Autónoma de la Universidad de Nuevo León; VIH: Virus de Inmunodeficiencia Humana.

Recientemente se sumó el Mavrilimumab a los estudios de investigación, un anticuerpo monoclonal humano que inhibe al receptor del factor estimulante de colonias de macrófagos y granulocitos humanos (GM-CSF-R), que recién en 2017 se determinó su eficacia en el 
Guzmán Valdivia Gómez, G.; Domínguez González, A. D.; Álvarez Rodríguez, S.; Meneses Ruiz, D. M.

tratamiento de la artritis reumatoide al inhibir la activación de los monocitos y macrófagos y, en consecuencia, la síntesis de citocinas. Sólo existen cuatro ensayos clínicos en fase II con Mavrilimumab, tres en Estados Unidos y uno en Italia, dirigidos a pacientes con neumonía severa e hiperinflamación sistémica. (De Luca et al., 2020)

\section{Discusión}

En las últimas dos décadas llevamos tres amenazas por coronavirus, esta última llegando al estado de pandemia. El brote de COVID-19 que vivimos es causado por un coronavirus conocido como SARS-CoV-2, que tiene similitudes con los coronavirus responsables del SARS y MERS.

Las estrategias más exitosas para tratar las enfermedades SARS y MERS fueron la reducción de la carga viral en etapas tempranas y el control de la respuesta inflamatoria, para el SARS se utilizó Ribavirina, Lopinavir y Ritonavir (LPV/r), Interferón tipo 1 (IFN), corticoesteroides, inmunoglobulinas intravenosa y plasma de pacientes convalecientes de SARS (Stockman et al., 2006). Para MERS, los tratamientos más efectivos fueron con Ribavirina e Interferón tipo 1 (Arabi et al., 2020). Los ensayos clínicos utilizados para tratar el COVID-19 iniciaron con criterios semejantes, pero debido a los resultados limitados mostrados en la eficacia y seguridad de los tratamientos se han reposicionado otros fármacos.

Hasta el momento, los resultados de diferentes ensayos clínicos han mostrado que en la etapa de gran replicación viral en el tracto respiratorio superior y el inicio de la lesión en el alveolo pulmonar (fases I y IIa) no se recomienda prescribir tratamiento alguno, excepto paracetamol para el control de síntomas como fiebre, cefalea y dolor muscular. Medicamentos como Arbidol, Oseltamivir, Ivermectina, Azitromicina, Nitazoxanida, Cloroquina e Hidroxiclororoquina, Factor de trasferencia, inmunoglobulina intravenosa e interferones, han mostrado resultados controversiales y algunos centros hospitalarios han limitado su uso, aunque se siguen estudiando (RECOVERY Trial, 2020). 
Los medicamentos con mayor eficacia para limitar el avance de la enfermedad a etapas de mayor afectación con la consecuente reducción en el tiempo de hospitalización y uso de terapias intensivas son: Remdesivir, Favipiravir, Lopinavir/Ritonavir, Colchicina, Baricitinib combinado con Remdesivir, Tocilizumab, Sarilumab, Anakinra, plasma de pacientes convalecientes y heparinas a diversas dosis (Jean et al., 2020, Giovane et al., 2020).

En un ensayo clínico controlado del grupo RECOVERY, auspiciado por la Universidad de Oxford, demostró que la Dexametasona a bajas dosis redujo la mortalidad en pacientes que requieren oxigenoterapia o ventilación mecánica (Horby et al., 2020).

México contribuye con 45 ensayos clínicos de reposicionamiento de fármacos, de acuerdo con la información registrada por ClinicalTrials.gov, un registro de ensayos clínicos administrado por la Biblioteca Nacional de Medicina de los Estados Unidos, la mayor base de datos de ensayos clínicos, con registros de más de 329,000 ensayos de 209 países.

Los fármacos antivirales que se están estudiando en México, solos o en combinación, son Azitromicina, Baricitinib, Cloroquina, Hidroxicloroquina, Ivermectina, Nitasoxanida, Remdesivir y Ruxolitinib. Los inmunomoduladores son Colchicina, Tocilizumab, plasma de pacientes convalecientes y vacuna BCG. Entre las principales instituciones participantes en el estudio de reposicionamiento de fármacos se encuentran los Institutos Nacionales de Salud, el INSaBi, el ISSSTE, el Hospital Universitario de la Universidad Autónoma de Nuevo León, el Hospital Naval de Alta Especialidad y el Hospital Central PEMEX.

El presente trabajo cuenta con limitaciones relacionadas con que sólo se revisaron los ensayos clínicos incluidos en el repositorio Clinical trials sobre medicamentos antivirales y inmunomoduladores, dejando de lado todos aquellos fármacos que se utilizan para tratar las complicaciones sistémicas derivadas de la enfermedad. Además, la mayoría de los ensayos clínicos continúan en etapa activa, por lo que no se cuenta con resultados concluyentes.

\section{Conclusiones}

Actualmente no existe un tratamiento eficaz contra el virus SARS-CoV-2, los centros hospitalarios continúan manejando la enfermedad en las etapas de complicación con medidas encaminadas a reducir los efectos sistémicos inducidos por este virus. Los ensayos clínicos relacionados con los medicamentos antivirales continúan fortaleciendo la estrategia de 
Guzmán Valdivia Gómez, G.; Domínguez González, A. D.; Álvarez Rodríguez, S.; Meneses Ruiz, D. M.

reposicionamiento de fármacos, ya sean solos o combinados. La investigación a nivel mundial sigue generando información valiosa que se actualiza constantemente, sin que se haya podido establecer hasta ahora el tratamiento efectivo y seguro de primera elección. Continuar con ensayos clínicos avalados por comités de investigación y de ética en investigación de los medicamentos actuales e investigación básica encaminados a elucidar los mecanismos fisiopatológicos propios de la enfermedad, contribuirá al reposicionamiento de los medicamentos existentes y diseño de nuevos fármacos para el tratamiento específico del COVID-19, además del desarrollo de vacunas para el SARS-CoV-2.

\section{Agradecimientos}

Los autores agradecen a las autoridades de la Facultad Mexicana de Medicina y a la Dirección de Posgrado e Investigación de la Universidad La Salle, México por su apoyo para la realización de este trabajo.

\section{Referencias}

Arabi, Y. M., Shalhoub, S., Mandourah, Y., Al-Hameed, F., Al-Omari, A., Al Qasim, E., Jose, J., Alraddadi, B., Almotairi, A., Al Khatib, K., Abdulmomen, A., Qushmaq, I., Sindi, A. A., Mady, A., Solaiman, O., Al-Raddadi, R., Maghrabi, K., Ragab, A., Al Mekhlafi, G. A., ... Fowler, R. (2020). Ribavirin and Interferon Therapy for Critically Ill Patients With Middle East Respiratory Syndrome: A Multicenter Observational Study. Clinical Infectious Diseases: An Official Publication of the Infectious Diseases Society of America, 70(9), 1837-1844. https://doi.org/10.1093/cid/ciz544

Bonam, S. R., Kaveri, S. V., Sakuntabhai, A., Gilardin, L., \& Bayry, J. (2020). Adjunct Immunotherapies for the Management of Severely Ill COVID-19 Patients. Cell Reports Medicine, 1(2), 100016. https://doi.org/10.1016/j.xcrm.2020.100016

Bulut, C., \& Kato, Y. (2020). Epidemiology of COVID-19. Turkish Journal of Medical Sciences, 50(SI-1), 563-570. https://doi.org/10.3906/sag-2004-172 
Calderón, J. M., Zerón, H. M., \& Padmanabhan, S. (2020). Treatment with Hydroxychloroquine vs Hydroxychloroquine + Nitazoxanide in COVID-19 patients with risk factors for poor prognosis: A structured summary of a study protocol for a randomised controlled trial. Trials, 21(1), 504. https://doi.org/10.1186/s13063-020$\underline{04448-2}$

Cai, Q., Yang, M., Liu, D., Chen, J., Shu, D., Xia, J., Liao, X., Gu, Y., Cai, Q., Yang, Y., Shen, C., Li, X., Peng, L., Huang, D., Zhang, J., Zhang, S., Wang, F., Liu, J., Chen, L., ... Liu, L. (2020). Experimental Treatment with Favipiravir for COVID-19: An Open-Label Control Study. Engineering (Beijing, China). https://doi.org/10.1016/j.eng.2020.03.007

Cantini, F., Niccoli, L., Matarrese, D., Nicastri, E., Stobbione, P., \& Goletti, D. (2020). Baricitinib therapy in COVID-19: A pilot study on safety and clinical impact. The Journal of Infection. https://doi.org/10.1016/j.jinf.2020.04.017

Cao, B., Wang, Y., Wen, D., Liu, W., Wang, J., Fan, G., Ruan, L., Song, B., Cai, Y., Wei, M., Li, X., Xia, J., Chen, N., Xiang, J., Yu, T., Bai, T., Xie, X., Zhang, L., Li, C., ... Wang, C. (2020). A Trial of Lopinavir-Ritonavir in Adults Hospitalized with Severe Covid-19. The New England Journal of Medicine, 382(19), 1787-1799. https://doi.org/10.1056/NEJMoa2001282

Channappanavar, R., Fehr, A. R., Vijay, R., Mack, M., Zhao, J., Meyerholz, D. K., \& Perlman, S. (2016). Dysregulated Type I Interferon and Inflammatory MonocyteMacrophage Responses Cause Lethal Pneumonia in SARS-CoV-Infected Mice. Cell Host \& Microbe, 19(2), 181-193. https://doi.org/10.1016/j.chom.2016.01.007

Chen, N., Zhou, M., Dong, X., Qu, J., Gong, F., Han, Y., Qiu, Y., Wang, J., Liu, Y., Wei, Y., Xia, J., Yu, T., Zhang, X., \& Zhang, L. (2020). Epidemiological and clinical characteristics of 99 cases of 2019 novel coronavirus pneumonia in Wuhan, China: A descriptive study. Lancet (London, England), 395(10223), 507-513. https://doi.org/10.1016/S0140-6736(20)30211-7

Coronaviridae Study Group of the International Committee on Taxonomy of Viruses. (2020).

The species Severe acute respiratory syndrome-related coronavirus: Classifying 2019-nCoV and naming it SARS-CoV-2. Nature Microbiology, 5(4), 536-544. https://doi.org/10.1038/s41564-020-0695-Z 
Guzmán Valdivia Gómez, G.; Domínguez González, A. D.; Álvarez Rodríguez, S.; Meneses Ruiz, D. M.

Corral, P., Corral, G., \& Diaz, R. (2020). Colchicine and COVID-19. Journal of Clinical Pharmacology. https://doi.org/10.1002/jcph.1684

Costanzo, M., De Giglio, M. A. R., \& Roviello, G. N. (2020). SARS-CoV-2: Recent Reports on Antiviral Therapies Based on Lopinavir/Ritonavir, Darunavir/Umifenovir, Hydroxychloroquine, Remdesivir, Favipiravir and Other Drugs for the Treatment of the New Coronavirus. Current Medicinal Chemistry. https://doi.org/10.2174/0929867327666200416131117

Cui, J., Li, F., \& Shi, Z.-L. (2019). Origin and evolution of pathogenic coronaviruses. Nature Reviews. Microbiology, 17(3), 181-192. https://doi.org/10.1038/s41579-018-0118-9

De Luca, G., Cavalli, G., Campochiaro, C., Della-Torre, E., Angelillo, P., Tomelleri, A. et al. (2020). GM-CSF blockade with mavrilimumab in severe COVID-19 pneumonia and systemic hyperinflammation: A single-centre, prospective cohort study. The Lancet Rheumatology, S2665991320301703. $\quad$ https://doi.org/10.1016/S26659913(20)30170-3

Fu, Y., Cheng, Y., \& Wu, Y. (2020). Understanding SARS-CoV-2-Mediated Inflammatory Responses: From Mechanisms to Potential Therapeutic Tools. Virologica Sinica. https://doi.org/10.1007/s12250-020-00207-4

Giovane, R. A., Rezai, S., Cleland, E., \& Henderson, C. E. (2020). Current pharmacological modalities for management of novel coronavirus disease 2019 (COVID-19) and the rationale for their utilization: A review. Reviews in Medical Virology, e2136. https://doi.org/10.1002/rmv.2136

Goker Bagca, B., \& Biray Avci, C. (2020). The potential of JAK/STAT pathway inhibition by ruxolitinib in the treatment of COVID-19. Cytokine \& Growth Factor Reviews. https://doi.org/10.1016/j.cytogfr.2020.06.013

Guan, W.-J., Ni, Z.-Y., Hu, Y., Liang, W.-H., Ou, C.-Q., He, J.-X., Liu, L., Shan, H., Lei, C.L., Hui, D. S. C., Du, B., Li, L.-J., Zeng, G., Yuen, K.-Y., Chen, R.-C., Tang, C.-L., Wang, T., Chen, P.-Y., Xiang, J., ... China Medical Treatment Expert Group for Covid-19. (2020). Clinical Characteristics of Coronavirus Disease 2019 in China. The New England Journal of Medicine, 382(18), 1708-1720. https://doi.org/10.1056/NEJMoa2002032 
Hasöksüz, M., Kiliç, S., \& Saraç, F. (2020). Coronaviruses and SARS-COV-2. Turkish Journal of Medical Sciences, 50(SI-1), 549-556. https://doi.org/10.3906/sag-2004$\underline{127}$

Horby PW et al. (2020). RECOVERY Trial press release. https://www.recoverytrial.net/results

Jean, S.-S., Lee, P.-I., \& Hsueh, P.-R. (2020). Treatment options for COVID-19: The reality and challenges. Journal of Microbiology, Immunology, and Infection = Wei Mian Yu Gan Ran Za Zhi, 53(3), 436-443. https://doi.org/10.1016/j.jmii.2020.03.034

Jin, Y., Yang, H., Ji, W., Wu, W., Chen, S., Zhang, W., \& Duan, G. (2020). Virology, Epidemiology, Pathogenesis, and Control of COVID-19. Viruses, 12(4). https://doi.org/10.3390/v12040372

Karimi-Zarchi, M., Neamatzadeh, H., Dastgheib, S. A., Abbasi, H., Mirjalili, S. R., Behforouz, A., Ferdosian, F., \& Bahrami, R. (2020). Vertical Transmission of Coronavirus Disease 19 (COVID-19) from Infected Pregnant Mothers to Neonates: A Review. Fetal and Pediatric Pathology, 1-5. https://doi.org/10.1080/15513815.2020.1747120

Lai, C.-C., Ko, W.-C., Lee, P.-I., Jean, S.-S., \& Hsueh, P.-R. (2020). Extra-respiratory manifestations of COVID-19. International Journal of Antimicrobial Agents, 106024. https://doi.org/10.1016/j.ijantimicag.2020.106024

Ledford, H. (2020). Coronavirus breakthrough: Dexamethasone is first drug shown to save lives. Nature, 582(7813), 469. https://doi.org/10.1038/d41586-020-01824-5

Liu, B., Li, M., Zhou, Z., Guan, X., \& Xiang, Y. (2020). Can we use interleukin-6 (IL-6) blockade for coronavirus disease 2019 (COVID-19)-induced cytokine release syndrome (CRS)? Journal of Autoimmunity, 111, 102452. https://doi.org/10.1016/j.jaut.2020.102452

Liu, Y.-C., Kuo, R.-L., \& Shih, S.-R. (2020). COVID-19: The First Documented Coronavirus Pandemic in History. Biomedical Journal. https://doi.org/10.1016/j.bj.2020.04.007

McMichael, T. M., Currie, D. W., Clark, S., Pogosjans, S., Kay, M., Schwartz, N. G., Lewis, J., Baer, A., Kawakami, V., Lukoff, M. D., Ferro, J., Brostrom-Smith, C., Rea, T. D., Sayre, M. R., Riedo, F. X., Russell, D., Hiatt, B., Montgomery, P., Rao, A. K., ... Duchin, J. S. (2020). Epidemiology of Covid-19 in a Long-Term Care Facility in King 
Guzmán Valdivia Gómez, G.; Domínguez González, A. D.; Álvarez Rodríguez, S.; Meneses Ruiz, D. M.

County, Washington. The New England Journal of Medicine. https://doi.org/10.1056/NEJMoa2005412

Mehta, P., McAuley, D. F., Brown, M., Sanchez, E., Tattersall, R. S., Manson, J. J., \& HLH Across Speciality Collaboration, UK. (2020). COVID-19: Consider cytokine storm syndromes and immunosuppression. Lancet (London, England), 395(10229), 10331034. https://doi.org/10.1016/S0140-6736(20)30628-0

Morawska, L., \& Cao, J. (2020). Airborne transmission of SARS-CoV-2: The world should face the reality. Environment International, 139, 105730. https://doi.org/10.1016/j.envint.2020.105730

Organización Mundial de la Salud (2020, 3 de junio). Recuperado de https://covid19. who.int/ Park, M., Thwaites, R. S., \& Openshaw, P. J. M. (2020). COVID-19: Lessons from SARS and MERS. European Journal of Immunology, 50(3), 308-311. https://doi.org/10.1002/eji.202070035

Prete, M., Favoino, E., Catacchio, G., Racanelli, V., \& Perosa, F. (2020). SARS-CoV-2 Inflammatory Syndrome. Clinical Features and Rationale for Immunological Treatment. International Journal of Molecular Sciences, 21(9). https://doi.org/10.3390/ijms21093377

Ranieri, V. M., Pettilä, V., Karvonen, M. K., Jalkanen, J., Nightingale, P., Brealey, D., Mancebo, J., Ferrer, R., Mercat, A., Patroniti, N., Quintel, M., Vincent, J.-L., Okkonen, M., Meziani, F., Bellani, G., MacCallum, N., Creteur, J., Kluge, S., ArtigasRaventos, A., ... INTEREST Study Group. (2020). Effect of Intravenous Interferon $\beta-1 \mathrm{a}$ on Death and Days Free From Mechanical Ventilation Among Patients With Moderate to Severe Acute Respiratory Distress Syndrome: A Randomized Clinical Trial. JAMA. https://doi.org/10.1001/jama.2019.22525

RECOVERY Trial, 2020. Randomised Evaluation of COVID-19 Therapy, en https://www.recoverytrial.net/results.

Rosa, S. G. V., \& Santos, W. C. (2020). Clinical trials on drug repositioning for COVID-19 treatment. Revista Panamericana De Salud Publica = Pan American Journal of Public Health, 44, e40. https://doi.org/10.26633/RPSP.2020.40 
Sanders, J. M., Monogue, M. L., Jodlowski, T. Z., \& Cutrell, J. B. (2020). Pharmacologic Treatments for Coronavirus Disease 2019 (COVID-19): A Review. JAMA. https://doi.org/10.1001/jama.2020.6019

Sanofi. Sanofi and Regeneron begin global Kevzara (sarilumab) clinical trial program in patients with severe COVID-19 (2020, marzo 18). Recuperado de http://news.sanofi.us/2020-03-16-Sanofi-and-Regeneron-begin-global-Kevzara-Rsarilumabclinical-trial-program-in-patients-with-severe-COVID-19

Sharun, K., Dhama, K., Patel, S. K., Pathak, M., Tiwari, R., Singh, B. R., Sah, R., BonillaAldana, D. K., Rodriguez-Morales, A. J., \& Leblebicioglu, H. (2020). Ivermectin, a new candidate therapeutic against SARS-CoV-2/COVID-19. Annals of Clinical Microbiology and Antimicrobials, 19(1), 23. https://doi.org/10.1186/s12941-020$\underline{00368-\mathrm{W}}$

Siddiqi, H. K., \& Mehra, M. R. (2020). COVID-19 illness in native and immunosuppressed states: A clinical-therapeutic staging proposal. The Journal of Heart and Lung Transplantation: The Official Publication of the International Society for Heart Transplantation, 39(5), 405-407. https://doi.org/10.1016/j.healun.2020.03.012

Stockman, L. J., Bellamy, R., \& Garner, P. (2006). SARS: Systematic review of treatment effects. PLoS Medicine, 3(9), e343. https://doi.org/10.1371/journal.pmed.0030343

Tay, H. S., \& Harwood, R. (2020). Atypical presentation of COVID-19 in a frail older person. Age and Ageing. https://doi.org/10.1093/ageing/afaa068

Vaduganathan, M., Vardeny, O., Michel, T., McMurray, J. J. V., Pfeffer, M. A., \& Solomon, S. D. (2020). Renin-Angiotensin-Aldosterone System Inhibitors in Patients with Covid-19. The New England Journal of Medicine, 382(17), 1653-1659. https://doi.org/10.1056/NEJMsr2005760

Villar, J., Ferrando, C., Martínez, D., Ambrós, A., Muñoz, T., Soler, J. A., Aguilar, G., Alba, F., González-Higueras, E., Conesa, L. A., Martín-Rodríguez, C., Díaz-Domínguez, F. J., Serna-Grande, P., Rivas, R., Ferreres, J., Belda, J., Capilla, L., Tallet, A., Añón, J. M., ... dexamethasone in ARDS network. (2020). Dexamethasone treatment for the acute respiratory distress syndrome: A multicentre, randomised controlled trial. The Lancet. Respiratory Medicine, 8(3), 267-276. https://doi.org/10.1016/S2213$\underline{2600(19) 30417-5}$ 
Guzmán Valdivia Gómez, G.; Domínguez González, A. D.; Álvarez Rodríguez, S.; Meneses Ruiz, D. M.

Walls, A. C., Park, Y.-J., Tortorici, M. A., Wall, A., McGuire, A. T., \& Veesler, D. (2020). Structure, Function, and Antigenicity of the SARS-CoV-2 Spike Glycoprotein. Cell, 181(2), 281-292.e6. https://doi.org/10.1016/j.cell.2020.02.058

Wang, M., Cao, R., Zhang, L., Yang, X., Liu, J., Xu, M., Shi, Z., Hu, Z., Zhong, W., \& Xiao, G. (2020). Remdesivir and chloroquine effectively inhibit the recently emerged novel coronavirus (2019-nCoV) in vitro. Cell Research, 30(3), 269-271. https://doi.org/10.1038/s41422-020-0282-0

Xu, X., Han, M., Li, T., Sun, W., Wang, D., Fu, B., Zhou, Y., Zheng, X., Yang, Y., Li, X., Zhang, X., Pan, A., \& Wei, H. (2020). Effective treatment of severe COVID-19 patients with tocilizumab. Proceedings of the National Academy of Sciences of the United States of America, 117(20), 10970-10975. https://doi.org/10.1073/pnas.2005615117

Yang, J., Zheng, Y., Gou, X., Pu, K., Chen, Z., Guo, Q., Ji, R., Wang, H., Wang, Y., \& Zhou, Y. (2020). Prevalence of comorbidities and its effects in patients infected with SARSCoV-2: A systematic review and meta-analysis. International Journal of Infectious Diseases: IJID: Official Publication of the International Society for Infectious Diseases, 94, 91-95. https://doi.org/10.1016/j.ijid.2020.03.017

Ye, Q., Wang, B., \& Mao, J. (2020). The pathogenesis and treatment of the 'Cytokine Storm' in COVID-19. The Journal of Infection, 80(6), 607-613. https://doi.org/10.1016/j.jinf.2020.03.037

Zeng, Q.-L., Yu, Z.-J., Gou, J.-J., Li, G.-M., Ma, S.-H., Zhang, G.-F., Xu, J.-H., Lin, W.-B., Cui, G.-L., Zhang, M.-M., Li, C., Wang, Z.-S., Zhang, Z.-H., \& Liu, Z.-S. (2020). Effect of Convalescent Plasma Therapy on Viral Shedding and Survival in COVID19 Patients. The Journal of Infectious Diseases. https://doi.org/10.1093/infdis/jiaa228 Zhang, X., Hasoksuz, M., Spiro, D., Halpin, R., Wang, S., Vlasova, A., Janies, D., Jones, L. R., Ghedin, E., \& Saif, L. J. (2007). Quasispecies of bovine enteric and respiratory coronaviruses based on complete genome sequences and genetic changes after tissue culture adaptation. Virology, 363(1), 1-10. 
COVID-19: fisiopatología y propuestas terapéuticas en investigación clínica

Zhou, D., Dai, S.-M., \& Tong, Q. (2020). COVID-19: A recommendation to examine the effect of hydroxychloroquine in preventing infection and progression. The Journal of Antimicrobial Chemotherapy. https://doi.org/10.1093/jac/dkaa114 